CLINICAL STUDY

\title{
Altered insulin requirement in patients with type 1 diabetes and primary adrenal insufficiency receiving standard glucocorticoid replacement therapy
}

\author{
Ulf Elbelt, Stefanie Hahner and Bruno Allolio \\ Endocrinology and Diabetes Unit, Department of Medicine I, University of Wuerzburg, Josef-Schneider-Strasse 2, 97080 Wuerzburg, Germany \\ (Correspondence should be addressed to U Elbelt who is now at Clinical Endocrinology, Department of Medicine, Gastroenterology, Hepatology and \\ Endocrinology, Charité Campus Mitte, Charité University Medicine Berlin, Charitéplatz 1, 10117 Berlin, Germany; Email: ulf.elbelt@charite.de)
}

\begin{abstract}
Objective: Current glucocorticoid replacement regimens fail to fully mimic physiologic cortisol secretion in patients with primary adrenal insufficiency. This may lead to changes in insulin requirement in patients with primary adrenal insufficiency and type 1 diabetes. Therefore, we assessed insulin requirement in patients with autoimmune polyendocrine syndrome type 2 (APS-2).

Design and subjects: Ten females with primary adrenal insufficiency and type 1 diabetes (mean duration of type 1 diabetes $13 \pm 11$ years and of primary adrenal insufficiency $11 \pm 9$ years) were retrospectively assessed regarding insulin regimen and insulin dose adjustment. Data were compared with control patients matched for age, sex and duration of diabetes drawn from all patients with type 1 diabetes attending the diabetes outpatient clinics at the University Hospital Wuerzburg for a scheduled consultation.

Results: Glycaemia was well controlled in both groups (mean $\mathrm{HbA1c} 6.99 \pm 0.81 \%$ in APS-2 patients versus $6.69 \pm 1.03 \%$ in control patients). The mean weight-adjusted daily dose of insulin was nonsignificantly higher in patients with APS-2 compared with control patients $(0.69 \pm 0.35 \mathrm{IU} / \mathrm{kg}$ body weight versus $0.51 \pm 0.17$ respectively). The mean insulin (IU)/carbohydrate-ratio for $10 \mathrm{~g}$ of carbohydrate in the morning was $1.9 \pm 1.0$ and $1.4 \pm 0.5$ respectively. However, the insulin/carbohydrate-ratios were significantly higher in the APS-2 patients both at noon (mean ratio $2.0 \pm 0.9$ vs $1.1 \pm 0.5$ in control patients) and in the evening (mean ratio $2.1 \pm 1.1$ vs $1.3 \pm 0.5$ respectively; $P<0.05)$.

Conclusions: Glucocorticoid replacement therapy in patients with primary adrenal insufficiency and type 1 diabetes leads to significant changes in insulin requirement compared with patients with type 1 diabetes only.
\end{abstract}

European Journal of Endocrinology 160 919-924

\section{Introduction}

Autoimmune polyendocrine syndrome type 2 (APS-2) is a rare disease with an incidence of 1.4-4.5 cases per 100000 population (1), affecting mainly females, the female/male ratio being 2.7-3.7 (2). It is characterised by the presence of primary adrenal insufficiency, autoimmune thyroid disease (in 69-82\%) and/or type 1 diabetes (in $30-52 \%)(3,4)$. The presentation of primary adrenal insufficiency with recurrent severe hypoglycaemia due to glucocorticoid deficiency in patients with type 1 diabetes has been well described (5-9), and a low threshold for investigating patients with type 1 diabetes to detect Addison's disease is advised $(10,11)$. An increase in insulin requirement to former levels and a reversal of hypoglycaemia following initiation of glucocorticoid replacement therapy are frequently mentioned in case reports (6-9). However, Burke \& Emanuel have also described the difficulty of glycaemic control in a young patient with type 1 diabetes and concomitant adrenal insufficiency due to a lability of glycaemia and exquisite insulin sensitivity (12).

Current glucocorticoid replacement regimens fail to fully mimic physiologic cortisol secretion and to restore the subjective health status to normal $(13,14)$. These limitations of glucocorticoid replacement may also impact on the insulin requirement in patients with APS-2. However, no information concerning problems of insulin therapy in patients with type 1 diabetes and concomitant adrenal insufficiency is available so far. We, therefore, for the first time, analysed the pattern of insulin requirement in a series of patients with APS-2 in detail and compared it to patients suffering from type 1 diabetes only. 


\section{Subjects and methods}

\section{Patients with APS-2}

We included in our retrospective analysis 10 patients with type 1 diabetes mellitus and primary adrenal insufficiency presently cared for as outpatients at the Endocrinology and Diabetes Unit of the University Hospital Wuerzburg $(n=8)$ or at a private practice setting in Berlin $(n=2)$. To identify these patients, we screened all patients with adrenal insufficiency registered at the University Hospital Wuerzburg $(n=149)$. Of this cohort eighty-three patients had primary adrenal insufficiency and eight of these patients also suffered from type 1 diabetes mellitus. In addition, two patients with APS-2 cared for at a private practice in Berlin by one of the authors (U E) were included. Adrenal insufficiency had been diagnosed at the time of clinical manifestation using the standard short corticotropin test (measurement of serum cortisol before and $60 \mathrm{~min}$ after i.v. administration of $250 \mu \mathrm{g} 1-24 \mathrm{ACTH})$. In all cases, peak serum cortisol concentrations after ACTH were $\ll 500 \mathrm{nmol} / \mathrm{l}$ and baseline plasma ACTH was invariably elevated (15). In the two patients with clinical manifestation of adrenal insufficiency before 1990, the diagnosis of adrenal insufficiency was verified by a review of the medical records. Patient data are given in Table 1. Physical examination and standard laboratory tests were performed during the scheduled consultation. Patients were invited to a separate interview to gather further information concerning the course of the illness, concomitant diseases, diabetesrelated complications, hypoglycaemia, insulin regimen and insulin dose adjustment. All patients were willing to participate and provided informed consent. The patients were female with a mean ( \pm s.D.) age of $44 \pm 15$ years (range 27-77). Mean disease duration was $13 \pm 11$ years (range 1-36) for type 1 diabetes and $11 \pm 9$ years (range 0.3-31) for primary adrenal insufficiency. In five women, type 1 diabetes occurred first followed later by primary adrenal insufficiency (range 0.3-24 years).
Three of them presented with severe hypoglycaemic episodes (range 1-10) prior to diagnosis. In these patients, the time from presentation of initial hypoglycaemia to the final diagnosis ranged from 3 to 48 months. The other five women developed primary adrenal insufficiency first followed later by type 1 diabetes (range 2-30 years). Eight of the patients suffered from concomitant autoimmune disease: lymphocytic thyroiditis (Hashimoto's thyroiditis; $n=6$ ), Graves' disease $(n=1)$, vitiligo $(n=1)$, premature ovarian failure $(n=1)$ and pernicious anaemia $(n=1)$ respectively. One patient had trisomy 21 as a predisposing condition. All patients with lymphocytic thyroiditis received replacement therapy with levothyroxine $\left(\mathrm{L}-\mathrm{T}_{4}\right.$; mean daily dose $100 \pm 41.8 \mu \mathrm{g}$, range 50-150), one patient in combination with $10 \mu \mathrm{g}$ liothyronine. The TSH of these patients was $1.08 \pm 0.84 \mathrm{mIU} / \mathrm{l}$ (range $0.33-2.29)$ with normal range of $0.3-4.0 \mathrm{mIU} / \mathrm{l}$. The patient suffering from Graves' disease had undergone subtotal thyroidectomy due to a relapse. She suffered from subclinical hypothyroidism (TSH $7.4 \mathrm{mIU} / \mathrm{l}$, free $\mathrm{T}_{4} 21.2 \mathrm{pmol} / \mathrm{l}$ (normal range 10.3-24.5)) under replacement therapy with $125 \mu \mathrm{g} \mathrm{L}_{-} \mathrm{T}_{4}$. The remaining three patients without thyroid disease had a mean TSH $0.63 \pm 0.14 \mathrm{mIU} / \mathrm{l}$ (range $0.47-0.72$ ) and peripheral free thyroid hormones within the normal range. For glucocorticoid replacement, eight women were taking hydrocortisone and two were taking cortisone acetate (1 mg hydrocortisone $=1.6 \mathrm{mg}$ cortisone acetate) (15). The administered daily dose was $24.7 \pm 5.0 \mathrm{mg}$ hydrocortisone (range 15.6-31.3 mg) and $0.40 \pm 0.13 \mathrm{mg}$ hydrocortisone/kg body weight (range 0.20-0.64). Glucocorticoid replacement was given as two (eight patients) or three daily doses (two patients); $61.4 \pm 9.6 \%$ (range $50.0-75.0 \%$ ) of the administered daily dose was taken in the morning, $64.3 \pm 8.5 \%$ by the patients with two and $50.0 \pm 0 \%$ by the patients with three daily doses. Patients were taking their first daily dose in the morning between 0700 and $0800 \mathrm{~h}$ and the second dose between 1330 and $1430 \mathrm{~h}$. The two patients with three daily doses took their third dose

Table 1 Sequence and age (in years) at presentation of autoimmune diseases in the patients with autoimmune polyendocrine syndrome type 2 (APS-2).

\begin{tabular}{llr}
\hline Age (years) & Sequence and age (years) at presentation & Hypoglycaemia $^{\text {a }}$ \\
\hline 29 & Type 1 diabetes (13), Graves' disease (15), adrenal insufficiency (29), vitiligo (duration unknown) & 4 \\
30 & Type 1 diabetes (12), adrenal insufficiency (24), lymphocytic thyroiditis (24) & 10 \\
45 & Type 1 diabetes (9), lymphocytic thyroiditis (29), adrenal insufficiency (33) & 1 \\
46 & Pernicious anaemia (35), type 1 diabetes (45), adrenal insufficiency (45) \\
56 & Type 1 diabetes (35), adrenal insufficiency (42), lymphocytic thyroiditis (42) \\
$27^{\mathrm{b}}$ & Lymphocytic thyroiditis (19), premature ovarian failure (19), adrenal insufficiency (21), type 1 \\
& diabetes (23) & \\
42 & Adrenal insufficiency (35), lymphocytic thyroiditis (35), type 1 diabetes (39) \\
43 & Adrenal insufficiency (25), lymphocytic thyroiditis (27), type 1 diabetes (28) \\
47 & Adrenal insufficiency (32), type 1 diabetes (36) \\
77 & Adrenal insufficiency (46), type 1 diabetes (76)
\end{tabular}

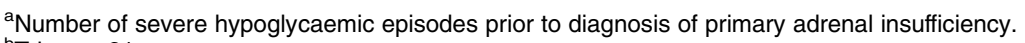

${ }^{\mathrm{b}}$ Trisomy 21. 
between 1800 and $2000 \mathrm{~h}$. None of the patients suffered from micro- or macrovascular chronic complications of diabetes mellitus.

\section{Patients with type 1 diabetes only}

Data from all patients $(n=56)$ with type 1 diabetes who attended the outpatient clinics of the Endocrinology and Diabetes Unit of the University Hospital Wuerzburg for a scheduled consultation with physical examination and routine laboratory testing during a 3-month period were screened. A careful history of the course of the illness, diabetes-related complications, hypoglycaemia, insulin regimen and insulin dose adjustments were available in all patients. Out of this cohort, control patients were selected for the patients with APS-2, matched for gender, age and duration of type 1 diabetes, prior to any further analysis (see Table 2). A regular thyroid function was present in all of these patients, mean TSH was 1.04 $\pm 0.64 \mathrm{mIU} / \mathrm{l}$ (range 0.18-2.40). The patient with reduced, but not suppressed, TSH showed peripheral free thyroid hormones within the normal range. One patient had mild non-proliferative diabetic retinopathy and mild gastroparesis and two patients had microalbuminuria without further signs of renal impairment.

Patients in both groups were in long-term care as outpatients at the Endocrinology and Diabetes Unit of the University Hospital Wuerzburg $(n=8)$ or at a private practice setting in Berlin $(n=2)$ by one of the authors. They had been repeatedly educated to balance the dose of prandial insulin with the amount of carbohydrates to be ingested. Therefore, carbohydrates are counted in grams and translated into 'carbohydrate choices'. Differing from the prevailing definition in the AngloAmerican literature (one carbohydrate choice equals $15 \mathrm{~g}$ carbohydrate) patients were educated that one carbohydrate choice equals $10 \mathrm{~g}$ carbohydrate, according to the guidelines of the German Diabetes Association. Prior to the separate interview of the patients with APS-2 and for the scheduled consultation of the patients with type 1 diabetes only, patients were

Table 2 Clinical data of patients with autoimmune polyendocrine syndrome type 2 (APS-2) and matched patients with type 1 diabetes only.

\begin{tabular}{lccc}
\hline & $\begin{array}{c}\text { APS-2 } \\
(n=10)\end{array}$ & $\begin{array}{c}\text { Control } \\
\text { patients } \\
(n=10)\end{array}$ & $\boldsymbol{P}$ \\
\hline Female $(n)$ & 10 & 10 & \\
Age (years) & $44 \pm 15$ & $43 \pm 15$ & $0.894^{\mathrm{a}}$ \\
Duration of diabetes (years) & $13 \pm 11$ & $13 \pm 11$ & $0.890^{\mathrm{a}}$ \\
BMl $\left(\mathrm{kg} / \mathrm{m}^{2}\right)$ & $24.1 \pm 5.3$ & $24.9 \pm 4.6$ & $0.722^{\mathrm{a}}$ \\
Patient with diabetes-related & 0 & 3 & $0.060^{\mathrm{b}}$ \\
$\quad$ complications $(n)$ & & & \\
\hline
\end{tabular}

Results are presented as mean \pm s.D., where appropriate.

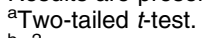

${ }^{b} \chi^{2}$ test. asked to record their plasma glucose measurements, their carbohydrate intake, and their insulin dose for several days (a minimum of 5 days). The mean insulin/carbohydrate-ratios for the morning, for noontime and the evening were then assessed. The mean total insulin dose (IU/day) and the mean basal insulin requirement (IU/day) were calculated.

\section{Laboratory measurements}

In all patients, $\mathrm{HbA1c}$ was measured by established methods (HPLC). Local HbA1c values were adjusted to diabetes control and complications trail (DCCT) standards (normal range 4.05-6.05\%) with an evaluated standardised procedure (16).

\section{Statistical analysis}

Distribution of the data was determined by using the Kolmogorov-Smirnov test. Results are expressed as means with S.D.s in parentheses for parametric data. Median and interquartile ranges are given for non-parametric data. The $\chi^{2}$ test (for categorical variables), two-tailed $t$-tests (for normally distributed unpaired data), Mann-Whitney $U$ test (for non-normally distributed data) and Pearson's coefficient of correlation were used for the statistical analyses. Changes at the 5\% probability level were considered statistically significant.

\section{Results}

\section{Metabolic control}

Measurement of $\mathrm{HbA} 1 \mathrm{c}$ revealed good glycaemic control in the majority of patients in both groups (Table 3). In patients with APS-2, HbA1c ranged from 5.9 to $8.2 \%$, in control patients from 5.6 to $8.2 \%(P=0.479)$.

Thyroid function was similar in patients with APS-2 (TSH $1.57 \pm 2.15$, range $0.33-7.40 \mathrm{mIU} / \mathrm{l})$ compared with control patients (TSH 1.04 \pm 0.64 , range 0.18-2.40; $P=0.472$ ).

\section{Insulin therapy}

In both groups, eight of the patients were treated with intensive conventional insulin therapy and two patients were treated with continuous s.c. insulin infusion (CSII) respectively. All patients with CSII used rapid-acting insulin analogues. Four patients with APS-2 used rapid-acting analogues in combination with long-acting analogues, two patients used rapid-acting analogues and intermediate-acting insulin, one patient used regular insulin alone due to extremely low insulin requirements, and the patient with trisomy 21 used rapid- and long-acting analogues and partially pre-mixed rapid-acting analogue with intermediateacting insulin. Five control patients used rapid-acting 
Table 3 Diabetes control, insulin therapy and hypoglycaemia in patients with autoimmune polyendocrine syndrome type 2 (APS-2) and control patients with type 1 diabetes only.

\begin{tabular}{|c|c|c|c|}
\hline & APS-2 $(n=10)$ & Control patients $(n=10)$ & $P$ \\
\hline \multicolumn{4}{|l|}{ Diabetes control } \\
\hline $\mathrm{HbA} 1 \mathrm{c}(\%)$ & $6.99 \pm 0.81$ & $6.69 \pm 1.03$ & $0.479^{\mathrm{a}}$ \\
\hline \multicolumn{4}{|l|}{ Insulin regimen } \\
\hline $\mathrm{ICIT}(n)$ & 8 & 8 & \\
\hline $\operatorname{CSII}(n)$ & 2 & 2 & \\
\hline \multicolumn{4}{|l|}{ Insulin preparation (ICIT) } \\
\hline \multicolumn{4}{|l|}{ Basal insulin } \\
\hline Long-acting analogue & 5 & 4 & \\
\hline Intermediate-acting insulin & 2 & 4 & \\
\hline None & 1 & 0 & $0.411^{\mathrm{b}}$ \\
\hline \multicolumn{4}{|l|}{ Prandial insulin } \\
\hline Rapid-acting analogue & 7 & 5 & \\
\hline Regular insulin & 1 & 3 & $0.248^{\mathrm{b}}$ \\
\hline \multicolumn{4}{|l|}{ Insulin requirement } \\
\hline Insulin (IU/day) & $44.5 \pm 20.5$ & $35.7 \pm 13.2$ & $0.271^{\mathrm{a}}$ \\
\hline Insulin (IU/day per kg body weight) & $0.69 \pm 0.35$ & $0.51 \pm 0.17$ & $0.159^{\mathrm{a}}$ \\
\hline Basal insulin requirement (IU/day) & $22.0 \pm 11.6$ & $18.4 \pm 8.3$ & $0.434^{\mathrm{a}}$ \\
\hline Basal insulin requirement (\% of daily dose) & $45.3 \pm 16.7$ & $51.1 \pm 12.1$ & $0.382^{\mathrm{a}}$ \\
\hline Correction factor (mg/dl blood glucose/IU insulin) & $40.0^{\mathrm{c}}$ & $40.0^{c}$ & $0.594^{\mathrm{d}}$ \\
\hline \multicolumn{4}{|l|}{ Hypoglycaemia } \\
\hline Severe episodes/year & $0.3^{\mathrm{c}}$ & $0.0^{c}$ & $0.531^{d}$ \\
\hline Without severe hypoglycaemia $(n)$ & 4 & 7 & $0.178^{\mathrm{b}}$ \\
\hline
\end{tabular}

Results are presented as mean \pm s.D., where appropriate. ${ }^{\mathrm{a}}$ Two-tailed $t$-test, ${ }^{\mathrm{b}} \chi^{2}$ test, ${ }^{\mathrm{c}}$ Median, ${ }^{\mathrm{d}}$ Mann-Whitney $U$-test

analogues to provide glycaemic coverage for meals, three in combination with intermediate-acting insulin and two with long-acting analogues. Three patients used regular insulin for glycaemic coverage of meals, two with long-acting analogues and one with intermediateacting insulin.

Insulin requirements are detailed in Table 3. The mean insulin/carbohydrate-ratio for $10 \mathrm{~g}$ of carbohydrate was $1.9 \pm 1.0$ (range 1-3.6) in the morning for the patients compared with $1.4 \pm 0.5$ (range 0.8-2.3) in the control patients $(P=0.191)$, and was significantly higher at noon with $2.0 \pm 0.9$ (range 1-3.3) vs $1.1 \pm 0.5$ (range $0-1.8 ; \quad P=0.018$ ) and also significantly higher in the evening with a mean of $2.1 \pm 1.1$ (range 1-4.6) vs $1.3 \pm 0.5$ (range $0.3-2.0 ; P=0.039$; Fig. 1). In both groups patients estimated their correction factor to be 40 , implying that one unit of insulin will decrease the blood glucose level by $40.0 \mathrm{mg} / \mathrm{dl}$ (APS-2: quartiles 28.8/40.0, control patients: quartiles $37.5 / 40.0 \mathrm{mg} / \mathrm{dl} ; P=0.594$; Table 3 ).

The two patients with APS-2 receiving three daily doses of glucocorticoid replacement had a $12 \%$ higher mean weight-adjusted daily dose of insulin (absolute difference: $0.08 \mathrm{IU} /$ day per kg body weight) compared with the patients with two daily doses of glucocorticoid replacement.

\section{Frequency of severe hypoglycaemia}

Six APS-2 patients and three control patients experienced severe hypoglycaemia (need of another person's assistance and blood glucose below $50 \mathrm{mg} / \mathrm{dl}$ or prompt recovery after oral carbohydrate, i.v. glucose or glucagon administration) during the last 3 years ( $\chi^{2}$ test: $P=0.178$ ). The median annual frequency of severe hypoglycaemia during the last 3 years was 0.3 (quartiles 0.0/0.4) in the patients with APS-2, and 0.0 (quartiles $0.0 / 1.0$ ) in the control patients $(P=0.531)$. All the patients (APS-2 and control patients) who experienced severe hypoglycaemia suffered from type 1 diabetes for at least 3 years and in the case of APS-2 suffered from adrenal insufficiency for at least 6 years. Therefore, hypoglycaemia prior to initiation of glucocorticoid replacement therapy was not a confounding factor.

When patients with APS-2 were asked for the predominating diabetes-related difficulties, six reported that they had significant difficulties with blood glucose management during physical activity.

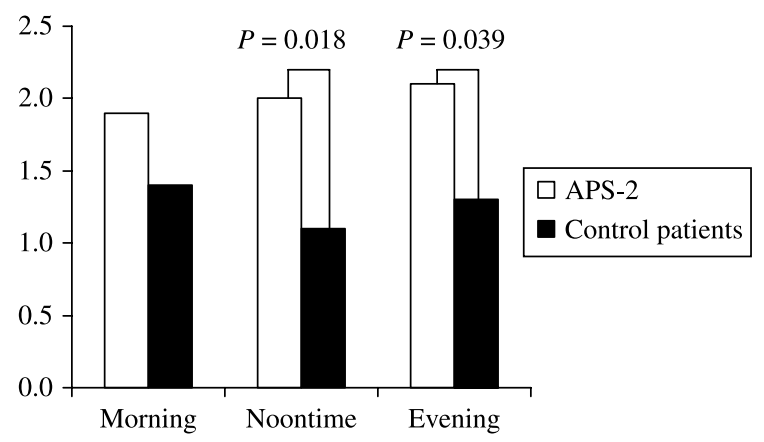

Figure 1 Mean insulin/carbohydrate-ratios (IU insulin/10 g of carbohydrate) for the morning, for noontime and for the evening derived from plasma glucose measurement, carbohydrate intake and insulin dose (minimum observational period 5 days). 


\section{Discussion}

The major finding of our study is a significantly different pattern in insulin requirement in patients with primary adrenal insufficiency and type 1 diabetes compared with matched control patients with type 1 diabetes only.

While the percentage of daily basal insulin coverage was lower in the patients with adrenal failure, insulin requirements to provide glycaemic coverage for meals were higher in patients with APS-2 compared with the control patients as evidenced by elevated insulin/ carbohydrate-ratios. While in individuals with type 1 diabetes and intact adrenal function the insulin requirement for prandial glycaemic coverage was the highest in the morning, in the patients with APS-2 receiving glucocorticoid replacement therapy the prandial insulin requirement increased throughout the day.

These differences most likely reflect differences in glucocorticoid availability. Current glucocorticoid replacement therapy is associated with two major problems, one is the total administered glucocorticoid dose and the other is the non-physiological distribution of oral glucocorticoids.

A daily oral administration of $15-25 \mathrm{mg}$ hydrocortisone is recommended for glucocorticoid replacement therapy (17). Usually, hydrocortisone is given in two or three daily doses, with one half to two-thirds in the morning (15). In our patients, the mean daily dose of hydrocortisone was $24.7 \mathrm{mg}$ and did not correlate to body mass index. There are still no established objective parameters for monitoring replacement therapy and for guiding dose adjustments. Instead, the evaluation of glucocorticoid replacement quality relies primarily on clinical judgement (18). When hydrocortisone replacement therapy was assessed by Peacey et al. in hypoadrenal patients with serum cortisol day curves, 24-h urine free cortisol excretion and markers of bone metabolism, a reduction in dose was conducted in $75 \%$ of the patients (19). The mean daily dose of hydrocortisone decreased from 29.5 to $20.8 \mathrm{mg}$.

The total daily insulin requirements adjusted to body weight in the patients with APS-2 were 35\% higher than in control patients with only type 1 diabetes. Tight glycaemic control was achieved in both groups showing mean $\mathrm{HbAlc}$ levels comparable with those of the intensive therapy DCCT cohort (20). The higher insulin requirement per body weight in the patients with APS-2 on replacement therapy and the significantly higher insulin requirement for prandial glycaemic coverage at noontime and in the evening compared with the control patients may indicate over replacement with glucocorticoids also in our cohort. However, while the mean hydrocortisone dose in our cohort is at the upper limit of the recommended dose range, it was not different from recently published data in a large series of patients with primary adrenal insufficiency (14).
The lower percentage of daily basal insulin coverage in the patients with adrenal failure and the distinct pattern of insulin requirement for prandial glycaemic coverage with an increase during the day also suggest pharmacokinetic limitations of standard hydrocortisone replacement therapy. This leads to abnormally lowcortisol levels during the night and in the early morning hours, whereas endogenous cortisol levels rise steeply from low levels at midnight to peak values around $0700 \mathrm{~h}$ in subjects with intact adrenals. Thus, insulin sensitivity is relatively high in the morning in patients with APS-2 but decreases after the ingestion of the morning dose of hydrocortisone leading to significantly higher insulin/carbohydrate-ratios throughout the day. Plat et al. showed a delayed appearance of relative insulin resistance after glucocorticoid administration in healthy volunteers in whom endogenous cortisol levels had been suppressed by treatment with metyrapone at 4-h-intervals (21). This effect was much more pronounced when $50 \mathrm{mg}$ hydrocortisone was administered in the late afternoon $(1700 \mathrm{~h})$ compared with administration in the early morning $(0500 \mathrm{~h})$. In these healthy volunteers insulin secretion rate increased for at least $12 \mathrm{~h}$, starting $4 \mathrm{~h}$ after hydrocortisone ingestion, to compensate for the observed rise in plasma glucose despite a significant decrease in insulin clearance in the evening. This most likely reflects an increase of insulin resistance after hydrocortisone treatment. These findings help to explain the significantly higher insulin/ carbohydrate-ratio in the evening in our patients. The usual administration of the second daily dose of glucocorticoid replacement in our patients between 1330 and $1430 \mathrm{~h}$ may lead to an increase of insulin resistance starting at about $1800 \mathrm{~h}$ which has to be covered by higher doses of prandial insulin in the evening.

Favourable effects of administration of modifiedrelease prednisone that adapts the release to the circadian rhythms of endogenous cortisol have been demonstrated in patients with rheumatoid arthritis (22). Modified-release preparations are under development also for long-term hydrocortisone substitution (23). Whether such formulations will allow a more physiological glucocorticoid replacement therapy in patients with adrenal insufficiency and lead to a normal pattern of insulin requirement in patients with concomitant diabetes type 1 has to be assessed.

In both groups, the annual frequency of severe hypoglycaemia was low. Still, there were more control patients without any severe hypoglycaemia. The course of severe hypoglycaemia in patients with both diabetes type 1 and adrenal insufficiency seems to be less favourable (24). An increase of catecholamines during hypoglycaemia and physical activity is a major component of hormonal counter regulation (25). An impaired counter regulatory hormone response due to inadequately low serum cortisol and epinephrine levels has been described by Phornphutkul et al. (7). 
Epinephrine synthesis in the adrenal medulla depends on high levels of local glucocorticoids stimulating the enzymatic activity of phenylethanolamine $\mathrm{N}$-methyltransferase. These local levels of glucocorticoids are not achieved by orally administered replacement therapy (26). The impaired epinephrine response may also explain the difficulties in glycaemic control during physical activity in our patients with APS-2 as it may facilitate activity related hypoglycaemia.

In summary, glucocorticoid replacement therapy in patients with primary adrenal insufficiency is associated with non-physiological glucocorticoid availability leading to significant changes in insulin requirements in patients with APS-2 as compared with patients with type 1 diabetes only. Special concern should be given to prevent over replacement with glucocorticoids in these patients and to identify adequate insulin/carbohydrateratios at noontime and in the evening.

\section{Declaration of interest}

The authors declare that there is no conflict of interest that would prejudice the impartiality of this scientific work.

\section{Funding}

This research did not receive any specific grant from any funding agency in the public, commercial or not-for-profit sector.

\section{References}

1 Betterle C, Volpato M, Greggio AN \& Presotto F. Type 2 polyglandular autoimmune disease. Journal of Pediatric Endocrinology and Metabolism 19969 113-123.

2 Betterle C \& Zanchetta R. Update on autoimmune polyendocrine syndromes (APS). Acta Bio Medica 2003 74 9-33.

3 Neufeld M, MaClaren NK \& Blizzard RM. Two types of autoimmune Addison's disease associated with different polyglandular autoimmune (PGA) syndromes. Medicine 198160 355-362.

4 Betterle C, Dal Pra C, Mantero F \& Zanchetta R. Autoimmune adrenal insufficiency and autoimmune polyendocrine syndromes: autoantibodies, autoantigens, and their applicability in diagnosis and disease prediction. Endocrine Reviews 200223 327-364.

5 Schroter W, Arends J, Runte L, Spelzhaus A, Neumeyer H, Talaulicar M, Boustani A \& Willms B. Hypoglycemia with loss of consciousness during insulin therapy as an initial symptom of Addison's disease. Report of 2 cases. Deutsche Medizinische Wochenschrift 1985110 840-842.

6 Armstrong L \& Bell PM. Addison's disease presenting as reduced insulin requirement in insulin dependent diabetes. BMJ 1996312 1601-1602.

7 Phornphutkul C, Boney CM \& Gruppuso PA. A novel presentation of Addison's disease: hypoglycemia unawareness in an adolescent with insulin-dependent diabetes mellitus. Journal of Pediatrics 1998 132 882-884.

8 McAulay V \& Frier BM. Addison's disease in type 1 diabetes presenting with recurrent hypoglycaemia. Postgraduate Medical Journal 200076 230-232.

9 Thomas JB, Petrovsky N \& Ambler GR. Addison's disease presenting in four adolescents with type 1 diabetes. Pediatric Diabetes 20045 207-211.

10 Brewer KW, Parziale VS \& Eisenbarth GS. Screening patients with insulin-dependent diabetes mellitus for adrenal insufficiency. New England Journal of Medicine 1997337202.
11 Likhari T, Magzoub S, Griffiths MJ, Buch HN \& Gama R. Screening for Addison's disease in patients with type 1 diabetes mellitus and recurrent hypoglycaemia. Postgraduate Medical Journal 200783 $420-421$.

12 Burke G \& Emanuel B. Addison's disease and diabetes mellitus in an adolescent. Clinical Pediatrics 19654 543-547.

13 Lovas K, Loge JH \& Husebye ES. Subjective health status in Norwegian patients with Addison's disease. Clinical Endocrinology $200256581-588$.

14 Hahner S, Loeffler M, Fassnacht M, Weismann D, Koschker AC, Quinkler M, Decker O, Arlt W \& Allolio B. Impaired subjective health status in 256 patients with adrenal insufficiency on standard therapy based on cross-sectional analysis. Journal of Clinical Endocrinology and Metabolism 200792 3912-3922.

15 Arlt W \& Allolio B. Adrenal insufficiency. Lancet $2003 \mathbf{3 6 1}$ 1881-1893.

16 Müller UA, Femerling M, Reinauer KM, Risse A, Voss M, Jörgens V, Berger M \& Mühlhauser I. Intensified treatment and education of type 1 diabetes as clinical routine. A nationwide quality-circle experience in Germany. ASD (the Working Group on Structured Diabetes Therapy of the German Diabetes Association). Diabetes Care 199922 B29-B34.

17 Kehlet H, Binder C \& Blichert-Toft M. Glucocorticoid maintenance therapy following adrenalectomy: assessment of dosage and preparation. Clinical Endocrinology 19765 37-41.

18 Arlt W, Rosenthal C, Hahner S \& Allolio B. Quality of glucocorticoid replacement in adrenal insufficiency: clinical assessment vs. timed serum cortisol measurements. Clinical Endocrinology 200664 384-389.

19 Peacey SR, Guo CY, Robinson AM, Price A, Giles MA, Eastell R \& Weetman AP. Glucocorticoid replacement therapy: are patients over treated and does it matter? Clinical Endocrinology 199746 $255-261$.

20 The Diabetes Control and Complications Trail Research Group. The effect of intensive treatment of diabetes on the development and progression of long-term complications in insulin-dependent diabetes mellitus. New England Journal of Medicine 1993329 977-986.

21 Plat L, Leproult R, L’Hermite-Baleriaux M, Fery F, Mockel J, Polonsky KS \& Van Cauter E. Metabolic effects of short-term elevations of plasma cortisol are more pronounced in the evening than in the morning. Journal of Clinical Endocrinology and Metabolism 199984 3082-3092.

22 Buttgereit F, Doering G, Schaeffler A, Witte S, Sierakowski S, Grominca-Ihle E, Jeka S, Krueger K, Szechinski J \& Alten R. Efficacy of modified-release versus standard prednisone to reduce duration of morning stiffness of the joints in rheumatoid arthritis (CAPRA-1): a double-blind, randomised controlled trail. Lancet $2008371205-214$.

23 Newell-Price J, Whiteman M, Rostami-Hodjegan A, Darzy K, Shalet S, Tucker GT \& Ross RJ. Modified-release hydrocortisone for circadian therapy: a proof-of-principle study in dexamethasonesuppressed normal volunteers. Clinical Endocrinology 200868 $130-135$.

24 Ajaz F, Kudva YC \& Erwin PJ. Residual dysphasia after severe hypoglycemia in a patient with immune-mediated primary adrenal insufficiency and type 1 diabetes mellitus: case report and systematic review of the literature. Endocrine Practice 200713 384-388.

25 Cryer PE. Diverse causes of hypoglycemia-associated autonomic failure in diabetes. New England Journal of Medicine 2004350 2272-2279.

26 Wurtman RJ \& Axelrod J. Control of enzymatic synthesis of adrenaline in the adrenal medulla by adrenal cortical steroids. Journal of Biological Chemistry 1966241 2301-2305.

Received 4 March 2009

Accepted 8 March 2009 